\title{
MHD SIMULATIONS OF CORONAL SUPRA-ARCADE DOWNFLOWS INCLUDING ANISOTROPIC THERMAL
} CONDUCTION

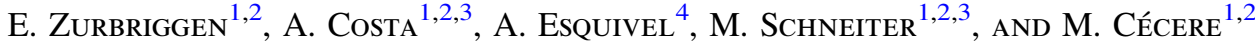 \\ ${ }^{1}$ Instituto de Investigaciones en Astronomía Teórica y Experimental (IATE), Córdoba, Argentina; ezurbriggen@unc.edu.ar, acosta@unc.edu.ar \\ ${ }^{2}$ Consejo Nacional de Investigaciones Científicas y Técnicas (CONICET), Argentina \\ ${ }^{3}$ Facultad de Ciencias Exactas, Físicas y Naturales, Universidad Nacional de Córdoba (UNC), Córdoba, Argentina \\ ${ }^{4}$ Instituto de Ciencias Nucleares, Universidad Nacional Autónoma de México, Mexico \\ Received 2016 July 29; revised 2016 September 23; accepted 2016 September 23; published 2016 November 18
}

\begin{abstract}
Coronal supra-arcade downflows (SADs) are observed as dark trails descending toward hot turbulent-fan-shaped regions. Due to the large temperature values and gradients in these fan regions, the thermal conduction (TC) should be very efficient. While several models have been proposed to explain the triggering and the evolution of SADs, none of these scenarios address a systematic consideration of TC. Thus, we accomplish this task numerically simulating the evolution of SADs within this framework. That is, SADs are conceived as voided (subdense) cavities formed by nonlinear waves triggered by downflowing bursty localized reconnection events in a perturbed hot fan. We generate a properly turbulent fan, obtained by a stirring force that permits control of the energy and vorticity input in the medium where SADs develop. We include anisotropic TC and consider plasma properties consistent with observations. Our aim is to study whether it is possible to prevent SADs from vanishing by thermal diffusion. We find that this will be the case, depending on the turbulence parameters, in particular if the magnetic field lines are able to envelope the voided cavities, thermally isolating them from the hot environment. Velocity shear perturbations that are able to generate instabilities of the Kelvin-Helmholtz type help to produce magnetic islands, extending the lifetime of SADs.
\end{abstract}

Key words: conduction - magnetohydrodynamics (MHD) - shock waves - Sun: corona - Sun: magnetic fields turbulence

Supporting material: animation

\section{INTRODUCTION}

The observational imprints of supra-arcade downflows (SADs) are dark (subdense) moving trails descending from $\sim 40-60 \mathrm{Mm}$ above the arcades, with downward speeds of $\sim 50-500 \mathrm{~km} \mathrm{~s}^{-1}$ and sizes of $\sim 1-10 \mathrm{Mm}$ (McKenzie \& Hudson 1999; Savage et al. 2012b; Innes et al. 2014). SADs have always been detected during long-term erupting flares associated with coronal mass ejections (CMEs; Liu et al. 2013). Furthermore, they have been observed immersed in hot turbulent current sheet (CS) regions, with temperatures $(T \sim 10 \mathrm{MK})$ much higher than the underlying coronal environment values (McKenzie 2013; Hanneman \& Reeves 2014), thus subject to strong gradients and large thermal conduction (TC) effects. Due to heat transfer, heat flowing from the high-temperature CS into the neighboring plasma causes an expansion of the region called thermal halo or fan (due to its shape).

In spite of the presence of large temperature gradients in SAD observations, TC effects are rarely considered explicitly in numerical simulations of fan regions where SADs develop (Costa et al. 2009; Linton et al. 2009; Guo et al. 2014). While several works on thermal effects in CS models have been developed (e.g., Yokoyama \& Shibata 2001; Bemporad 2008; Seaton \& Forbes 2009), to the best of our knowledge, none of the numerical scenarios proposed to explain the SADs' origin and dynamics address the systematic inclusion of TC.

Within such a framework, we proposed that SADs are voided cavities triggered by bursty reconnection events that occur during longer-term reconnection processes (Costa et al. 2009) in inhomogeneous fans (Cécere et al. 2012; see also Cécere et al. 2015, hereafter Paper I). A bursty reconnection event deposits energy in a localized and instantaneous fashion, thus producing a blast wave. The blast generates shocks and rarefaction waves, leading to the formation of the subdense cavity. These reconnection events were considered as local processes, i.e., independent of the overall fan region, that can be triggered outside the fan. For simplicity, the current simulations assume that SADs are already immersed inside the fan. The bursty reconnection events are simulated as instantaneous pressure pulses.

TC is a sensitive function of the temperature $\left(\propto T^{5 / 2}\right)$ and is highly anisotropic in the presence of magnetic fields (Spitzer 1962), where the heat flux is mostly funneled along the field direction. Therefore, in an environment with magnetic fields and large thermal gradients, TC is highly efficient along the magnetic field lines, but almost null in the perpendicular direction. For this reason, almost closed magnetic field structures are left as the only efficient mechanism to hinder TC. In this setting, Pagano et al. (2007) studied the evolution of CME cores embedded in a hot coronal plasma using the magnetic field as thermal insulation to suppress TC.

In the context of the SADs and their environment, Paper I showed that TC can only be neglected if the fan densities and the characteristic longitudes are large enough. This assumption implies a fan where $\beta>1$ (the ratio of thermal pressure to magnetic pressure). Also, McKenzie (2013) and Scott et al. (2016) found relatively large values of $\beta$ in the corona $(>1)$, suggesting either that the density and/or the temperature are larger than generally assumed, or that the magnetic field is small enough. Thus, the fine determination of the plasma parameters in SADs' environment is a key question when trying to solve the importance of TC in this context, as well as the configuration of the magnetic field, since it might behave as 
Table 1

Simulated-stages Parameters

\begin{tabular}{lcccccc}
\hline \hline Stages & $T(\mathrm{MK})$ & $\rho\left(\mathrm{gr} \mathrm{cm}^{-3}\right)$ & $\boldsymbol{B} \cdot \hat{\boldsymbol{j}}(\mathrm{G})$ & $|\boldsymbol{v}|\left(c_{s}\right)$ & Stir & TC \\
\hline Turb & 6.0 & $1.2 \times 10^{-14}$ & 3.0 & 0.0 & yes & no \\
Fan & $\langle 7.0\rangle$ & $\left\langle 1.2 \times 10^{-14}\right\rangle$ & $\langle 3.0\rangle$ & $\langle 0.21\rangle$ & no & no \\
\hline
\end{tabular}

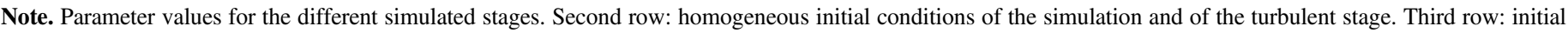

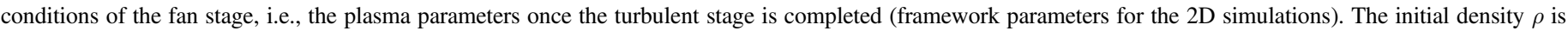
equivalent to $n_{i}=5 \times 109 \mathrm{~cm}^{-3}$. The quantities in $<\ldots>$ indicate averages over all grid cells. At the initial fan stage $<c_{s}>=4.0 \times 10^{7} \mathrm{~cm} \mathrm{~s}$.

a thermal insulator, allowing SADs to last longer (e.g., see Figure 4 of Asai et al. 2004).

Thus, as TC modifies the flow dynamics when its timescale is comparable to (or smaller than) the Alfvén timescale, models based on the development of instabilities, as proposed by Guo et al. (2014), require a thorough discussion of TC effects. In this sense, Hensler \& Vieser (2002) studied the suppression of hydrodynamic instability due to TC.

In what follows, the scenario proposed in Paper I is revised, taking into account the effects of anisotropic TC in a turbulent magnetized fan, i.e., the environment where SADs are observed. To summarize, the aim of this study is to address the following questions: how is it possible that SADs resist the thermal diffusion and are observed in hot fan regions? In fact, perturbations associated with typical SADs -with sizes of several megameters, typical coronal ion number densities of $\sim 10^{9} \mathrm{~cm}^{-3}$, and temperatures as high as 7-13 MK-will fade away in times (of a few seconds) that are at least two orders of magnitude smaller than a typical SAD lifetime. Is there a mechanism to avoid the thermal diffusion? The model is presented in Section 2, followed by a description of the simulations in Section 3. The results can be found in Section 4, and in Section 5 we provide our conclusions.

\section{THE MODEL}

We consider MHD equations in conservative form (CGS units):

$$
\begin{gathered}
\frac{\partial \rho}{\partial t}+\nabla \cdot(\rho \boldsymbol{v})=0 \\
\frac{\partial \rho \boldsymbol{v}}{\partial t}+\nabla \cdot(\rho \boldsymbol{v} \boldsymbol{v}-\boldsymbol{B B})+\nabla p_{*}=\boldsymbol{f}_{\mathrm{force}}, \\
\frac{\partial E}{\partial t}+\nabla \cdot\left[\left(E+p_{*}\right) \boldsymbol{v}-\boldsymbol{B}(\boldsymbol{v} \cdot \boldsymbol{B})+\boldsymbol{F}_{c}\right]=0, \\
\frac{\partial \boldsymbol{B}}{\partial t}=\nabla \times(\boldsymbol{v} \times \boldsymbol{B}) \\
E=\frac{1}{2} \rho v^{2}+e+\frac{B^{2}}{2} \\
p_{*}=p+\frac{B^{2}}{2}, \\
p=(\gamma-1) e=\frac{R}{\bar{\mu}} \rho T
\end{gathered}
$$

where $\rho$ is the mass density, $t$ is the time, $\boldsymbol{v}$ is the plasma flow velocity, $p$ is the thermal pressure, $\boldsymbol{B}$ is the magnetic field divided by $\sqrt{4 \pi}, E$ is the total energy, $e$ is the internal (thermal) energy, $\gamma=5 / 3$ is the ratio of the specific heats $\left(C_{P} / C_{V}\right), R$ is the gas constant, $\boldsymbol{F}_{c}$ is the heat flux, and $\boldsymbol{f}_{\text {force }}$ is a turbulence driving force. The plasma is assumed to be completely ionized with solar abundance. ${ }^{5}$ Hence, the density is $\rho=\left(n_{i}+n_{e}\right) m_{\mathrm{H}} \bar{\mu}$, where $n_{i}$ is the ion numerical density, $n_{e}=\bar{z} n_{i}$ is the free electron numerical density, $\bar{\mu}=0.613$ is the mean atomic mass, $\bar{z}=1.445$ is the mean atomic number, and $m_{\mathrm{H}}$ is the hydrogen atomic mass.

Since here we are interested in the relation between SADs and TC in the framework of the scenario proposed in previous papers (Costa et al. 2009; Cécere et al. 2012; Paper I), to perform the runs, we assume a set of characteristic parameters that lead to subdense cavities affected by TC when a pressure pulse in a 2D background turbulent fan is imposed. The set of values is given in Table 1. Also, Savage et al. (2012b) showed that for a fan with temperatures in the range of $\sim 10-13 \mathrm{MK}$ the $\mathrm{SAD}$ emission measure (EM) contrast has to be at least a factor of two with respect to the surroundings (see Figure 4 of their paper).

Based on these observational constraints, and in order to numerically reproduced a detectable $\mathrm{SAD}$, we know that the following conditions have to be satisfied: (1) an EM contrast between the subdense cavity and its background of at least a factor of 4, and (2) this subdense cavity must last at least 1 minute in the turbulent background.

To calculate the EM, we make use of the approximation of optically thin plasmas,

$$
\mathrm{EM}=\int_{l_{i}}^{l_{e}} n_{e}^{2} d l .
$$

The EM quantifies the squared electronic density $n_{e}$ along the line-of-sight direction $\boldsymbol{l}$ of the observer. In what follows, the line of sight is assumed to be along the $z$-direction. As we perform 2D simulations in the $(x, y)$ plane, the EM evaluation is $\mathrm{EM} /\left(z_{e}-z_{i}\right)=n_{e}^{2}$, where we assume that the spatial dependence of the number density is $n_{e}(x, y)$. In that sense the EM that we present can be considered as the EM per unit length along the line of sight.

Some useful characteristic timescales can be calculated in order to estimate the relative importance of various physical processes in a typical fan. The conduction, radiative, and magnetic reconnection timescales are

$$
\begin{aligned}
t_{\mathrm{cond}} & =9.1 \times 10^{13} \frac{\rho L^{2}}{T^{2.5}}, \quad t_{\mathrm{rad}}=\frac{3}{2} \frac{P}{\bar{z} n_{i}^{2} E_{r}}, \\
t_{\mathrm{rec}} & =\frac{L}{v_{\mathrm{A}} M_{\mathrm{A}}^{2}},
\end{aligned}
$$

where $L$ is a characteristic length and $E_{r}$ is the radiative loss function $\left(E_{r} \simeq 4 \times 10^{-23} \mathrm{erg} \mathrm{cm}^{3} \mathrm{~s}^{-1}\right.$ for $T \simeq 10^{7} \mathrm{~K}$;

\footnotetext{
5 Solar abundance: $70.7 \% \mathrm{H}+27.4 \% \mathrm{He}+1.9 \%$ heavier elements (Prialnik 2000).
} 
Aschwanden 2005). For the reconnection timescale we assume that the reconnection is mediated by turbulence, following Lazarian \& Vishniac (1999), where $v_{\mathrm{A}}$ is the Alfvén speed and $M_{\mathrm{A}}$ is the Mach Alfvén number. When considering the set of plasma parameters of the current problem, the corresponding timescales are $t_{\text {cond }} \sim 0.5 \mathrm{~s}, t_{\text {rad }} \sim 10^{3} \mathrm{~s}$, and $t_{\text {rec }} \sim 10^{3} \mathrm{~s}$. Note that, except for $t_{\text {cond }}$, the timescales are of the same order of magnitude as a typical SAD lifetime, i.e., only the TC is capable of rapid modification of the SAD observability. SADs maintain a pressure balance with their neighborhood because they are hotter than the surrounding plasma; hence, unless a mechanism acts to inhibit the strong TC diffusion, SADs will not last for times comparable with the observations.

Estimations of the Kelvin-Helmholtz (KH) and RayleighTaylor (RT) instabilities are given by

$$
t_{\mathrm{KH}-\mathrm{RT}} \equiv \frac{\gamma}{\gamma-1} \frac{c_{s}}{g_{\text {Sun }}}
$$

or

$$
\tau_{\mathrm{KH}-\mathrm{RT}} \equiv \frac{\gamma}{\gamma-1} \frac{d c_{s}}{v^{2}},
$$

where $c_{S}$ is the sound velocity, $g_{\text {Sun }}$ is the solar gravitational acceleration at the low corona, and $d$ and $v$ are typical SAD widths and speeds. Equation (10) gives the timescale taking into account the Sun gravity (Chen \& Lykoudis 1972). Within the set of our framework parameters $t_{\mathrm{KH}-\mathrm{RT}} \sim 3 \times 10^{3} \mathrm{~s}$; thus, the conduction terms will diffuse the structures formed by these type of instabilities, making them unlikely to be detectable as SADs. On the other hand, Guo et al. (2014) generated SADs by means of Rayleigh-Taylor-type instabilities produced by reconnection downflows that exert a ram pressure between a lighter plasma above and a denser fan region below. In this case, Equation (11) gives the timescale taking into account an acceleration $v^{2} / d$ that plays the role of gravity. Guo et al. (2014) obtained SADs of widths $d \sim 5-12 \mathrm{Mm}$ and average speeds of $50 \mathrm{~km} \mathrm{~s}^{-1}$, leading to a $\tau_{\mathrm{KH}-\mathrm{RT}} \sim 10^{3} \mathrm{~s}$. Therefore, the (isotropic) TC would also produce a strong diffusion in structures formed by these types of instabilities. However, $\tau_{\text {KH-RT }}$ could be reduced considering larger and also typical SAD speeds $v$; additionally, SADs could be thermally isolated by their particular magnetic field configuration. In Paper I we argued that the source and sink terms (i.e., TC, radiation losses, and reconnection contribution of heat) could be ignored if they compensate each other. To show that this could be the case, we considered somewhat large values of the number density, $n_{i}=2 \times 10^{10} \mathrm{~cm}^{-3}$, and of the characteristic longitude $L=12 \mathrm{Mm}$. However, observational data also report SAD sizes of $\sim 1 \mathrm{Mm}$ that remain visible for several minutes, which cannot be reproduced within our model including TC, because their $t_{\text {cond }}$ are even shorter (see the discussion of Figure 1).

The bursty reconnection events are simulated as instantaneous pressure pulses. They are generated as sudden thermal pressure increases, not modifying the density at the pulse region. Consequently, the SAD temperature results are larger than its neighborhood values, allowing an almost total pressure equilibrium between the SAD structure and its near neighborhood. Our results from two-dimensional simulation seem to disagree with McKenzie \& Savage (2009), who suggested that the SAD collapse is avoided due to higher internal values of the magnetic pressure, but we need three-dimensional simulation to confirm this conclusion. Due to the simplified SAD setup proposed here (where the medium outside the fan is not modeled), we can only consider the situation where SADs are triggered already inside the fan. Hanneman \& Reeves (2014) pointed out that there is little evidence that SADs are hotter than the fan, but they are always hotter than the external background medium. Definitely a whole magnetic configuration (considering the fan and the background medium), with SADs generated outside and descending into the fan, might result in SADs with internal temperatures higher than the background medium but colder than the fan temperature values.

The purpose of the present work is to investigate whether different combinations of the parameters $(\rho, T, L)$, i.e., lower values of the density and smaller SAD sizes, together with additional physical mechanisms that prevent the action of the heat diffusion terms, could explain the observed SAD characteristics. That is, considering the high efficiency of the $\mathrm{TC}$ in the fan, we explore situations where the magnetic field lines could envelope an SAD, providing thermal insulation from its surroundings.

\section{NUMERICAL CODE AND INITIAL CONDITIONS}

To carry out the numerical simulations, we use the FLASH code (Fryxell et al. 2000, release 4.2.2) that solves the compressible MHD equations. We choose for our simulations the unsplit staggered mesh algorithm (Lee et al. 2009) available in FLASH, which employs a finite volume method with a directionally unsplit data reconstruction and the constraint transport method to enforce the $\nabla \cdot \boldsymbol{B}=0$ condition. The Riemann problems at the computational interfaces are calculated using the second-order Roe-type Riemann solver along with an MC slope limiter. A uniform Cartesian 2D grid with $300^{2}$ cells is used. The physical domain representing the fan is set up to $(15,15) \mathrm{Mm}$, with the $y$-coordinate pointing away (radially from the Sun, neglecting the curvature of its surface) and the $x$-coordinate parallel to the surface of the Sun. Periodic conditions are considered at all boundaries.

The preparation of the fan setup where the SADs are triggered is performed in two stages. Starting from homogeneous initial conditions given in the second row of Table 1, we generate a turbulent state. This stage is obtained by adding a driving force that generates a turbulent state, as is described in the next section. At the end of the stirring stage, the system reaches a Kolmogorov-like spectrum, with the plasma parameters shown in the third row of Table 1. This turbulent state is the initial condition of the second (fan) stage. During the fan stage, we turn off the stirring force, leaving the turbulence to gradually decay, but we also turn on the anisotropic TC, as well as a shear resembling the action of outflows coming from above the fan. After this stage, we impose four bursty reconnection pulses. The turbulent stage occupies the time interval $t_{\text {turb }}=0-300 \mathrm{~s}$ while, the fan stage takes 100 additional seconds $\left(t_{\text {fan }}=300-400 \mathrm{~s}\right)$. The shear perturbation is instantaneously applied in the $y$-component of the velocity in the form

$$
v_{y}(t, x, y)= \begin{cases}v_{y}(t, x, y)-v_{\mathrm{sh}} & \text { if } x<0, \\ v_{y}(t, x, y) & \text { if } x \geqslant 0,\end{cases}
$$




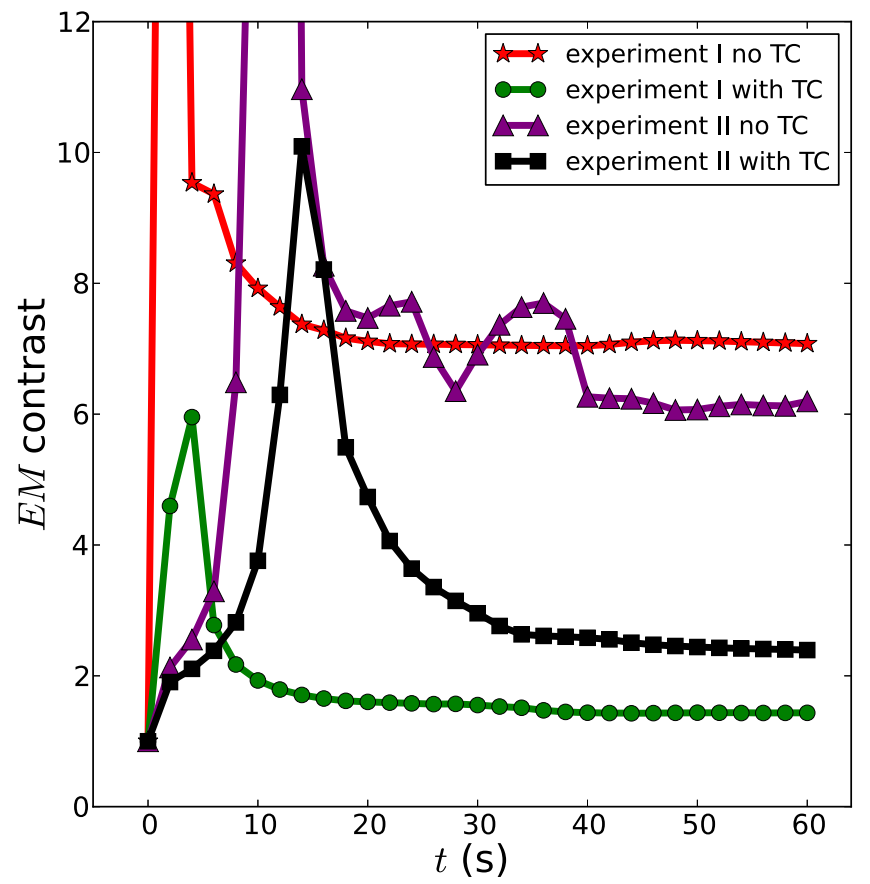

Figure 1. EM contrast against time for the blast experiments I and II with and without anisotropic TC.

at time $t=303.8 \mathrm{~s}$ and with $v_{\mathrm{sh}}=1.5 \times 10^{7} \mathrm{~cm} \mathrm{~s}^{-1}$. Finally, the four bursty reconnection pulses are instantaneously applied at time $t=305.9 \mathrm{~s}$, with diameters $L=1.2 \mathrm{Mm}$, labeled as $(A)$, $(B), \quad(C), \quad(D)$, centered at $\left(-3.0 \times 10^{8},-4.9 \times 10^{8}\right) \mathrm{cm}$, $\left(-9.6 \times 10^{6},-3.1 \times 10^{8}\right) \mathrm{cm},\left(5.3 \times 10^{7}, 5.5 \times 10^{8}\right) \mathrm{cm}$, and $\left(5.1 \times 10^{8}, 4.3 \times 10^{8}\right) \mathrm{cm}$, and with pressure contrasts of $\Delta P / P=(5,4,5,4)$, respectively.

In order to include the anisotropic TC (see, e.g., Spitzer 1962), a new module in the FLASH code was developed. In this new module the advected energy flux and the heat flux $\boldsymbol{F}_{c}$ were combined into a new energy flux inside the divergence on the left-hand side of Equation (3). The components of the anisotropic thermal flux $\boldsymbol{F}_{c}$ depend on the relative direction between the magnetic field and the temperature gradient as follows:

$$
\begin{gathered}
\boldsymbol{F}_{c}=-q_{\mathrm{eq}_{\|}} \nabla T_{\|}-q_{\mathrm{eq}_{\perp}} \nabla T_{\perp}, \\
\nabla T_{\|}=(\hat{\boldsymbol{b}} . \nabla T) \hat{\boldsymbol{b}}, \quad \nabla T_{\perp}=(\nabla T-\hat{\boldsymbol{b}} . \nabla T) \hat{\boldsymbol{b}}, \\
q_{\mathrm{eq}_{\|}}=\left(\frac{1}{q_{\mathrm{spi}_{\|}}}+\frac{1}{q_{\mathrm{sat}_{\|}}}\right)^{-1}, \\
q_{\mathrm{eq}_{\perp}}=\left(\frac{1}{q_{\mathrm{spi}_{\perp}}}+\frac{1}{q_{\mathrm{sat}_{\perp}}}\right)^{-1}, \\
q_{\mathrm{spi}_{\|}}=6.4 \times 10^{-7} T^{2.5}, \quad q_{\mathrm{sat}_{\|}}=\frac{5 \phi \rho c_{s}^{3}}{\left|\nabla T_{\|}\right|}, \\
q_{\mathrm{spi}_{\perp}}=4.6 \times 10^{32} \frac{\rho^{2}}{B^{2} \sqrt{T}}, \quad q_{\mathrm{sat}_{\perp}}=\frac{5 \phi \rho c_{s}^{3}}{\left|\nabla T_{\perp}\right|},
\end{gathered}
$$

where the subscripts $\|$ and $\perp$ refer to the directions along and across the magnetic field, respectively. $\nabla T$ is the temperature gradient, $\phi$ is a parameter that we set to $\phi=0.3, q_{\mathrm{spi}_{\|}}$and $q_{\mathrm{spi}_{\perp}}$ are the classical thermal conductivity (Spitzer 1962), $q_{\mathrm{sat} /}$ and $q_{\text {sat } \perp}$ are the saturated thermal conductivity (Cowie \& McKee 1977), and $q_{\mathrm{eq}_{\|}}$and $q_{\mathrm{eq}_{\perp}}$ are the equivalent thermal conductivity resulting from applying a harmonic flux limit.

TC imposes a strong diffusive constraint to the time steps. The constraint is a parabolic-like one, $\Delta t_{\text {diff }}<0.5 \rho C_{V} \Delta x^{2} /$ $\max \left(q_{\mathrm{eq}_{\|}}, q_{\mathrm{eq}_{\perp}}\right)$, where $C_{V}=R /((\gamma-1) \bar{u})$ is the specific heat at constant volume and $\Delta x$ is the cell size. Therefore, during the simulation runtime each time-step value is determined by the minimum between the usual CFL constraint and the diffusive constraint, i.e., $\Delta t=\min \left(\Delta t_{\mathrm{CFL}}, \Delta t_{\text {diff }}\right)$. The relatively low temperature value chosen for the initial fan stage $(T=7 \mathrm{MK})$ is a compromise between typical observational values and the computationally demanding diffuse constraint, $\Delta t_{\text {diff }}$.

Although for all cases explored (i.e., the parameter ranges $\left.\rho \sim 10^{-14}-10^{-15} \mathrm{~g} \mathrm{~cm}^{-3}, T \sim 1-10 \mathrm{MK},|\boldsymbol{B}| \sim[3-6] \mathrm{G}\right)$ the equivalent thermal conductivities always satisfied the condition $q_{\mathrm{eq}_{\|}} \gg q_{\mathrm{eq}_{\perp}}$, both contributions were taken into account.

\subsection{The Role of TC}

To illustrate the TC effects over a subdense cavity, we carry out a simple 2D MHD blast test, neglecting and including anisotropic TC. We use a $200^{2}$ cell setup. The initial density and pressure are homogeneous, except in a central circle of diameter $L$, where the pressure is increased by $\Delta P$. The fluid is initially at rest. A uniform magnetic field is assumed along the $y$-direction. In Figure 1, we show the results of two numerical experiments:

1. Our present framework parameters, based on observations of long-lived small SADs: $\rho=1.2 \times 10^{-14} \mathrm{~g} \mathrm{~cm}^{-3}, T=$ $7 \mathrm{MK}, \boldsymbol{B} \cdot \hat{\boldsymbol{j}}=3 \mathrm{G}, \boldsymbol{v}=0, L=1.2 \mathrm{Mm}$, and $\Delta P / P=5$.

2. With the parameter range explored in Paper I: $\rho=$ $5 \times 10^{-14} \mathrm{~g} \mathrm{~cm}^{-3}, \quad T=10 \mathrm{MK}, \quad \boldsymbol{B} \cdot \hat{\boldsymbol{j}}=5 \mathrm{G}, \quad \boldsymbol{v}=0$, $L=12 \mathrm{Mm}$ and $\Delta P / P=4$.

Both setups lead to a blast that generates a subdense cavity formed by shocks and expansion waves, which is faded away by the anisotropic TC. We define the EM contrast function, at a given time, as the maximum ratio between the background EM (almost equal to the unperturbed EM value) and its value inside the voided cavity, i.e., the EM $\operatorname{contrast}(t)=\max \left[\mathrm{EM}_{\text {back }} / \mathrm{EM}_{\text {cavity }}(t)\right]$, whose evolution can be analyzed in view of the TC timescale. This function gives a rough estimation of the permanence of the EM contrast along the time. Figure 1 shows the EM contrast as a function of time for both numerical experiments (I and II) with and without TC. Note that for large times the EM contrast $(t)>4$ for both cases that do not include TC (red and purple lines), meaning that an SAD is detectable according to the constraints given above. However, SADs will not be detected when TC is considered (green and black lines). In spite of this, the case equivalent to Paper I, with large values of $L$ and $\rho$, is in the limit of detection possibilities.

\subsection{Forced Turbulence}

Motivated by observational reports of the turbulent flows and vortices in fans (McKenzie 2013; Scott et al. 2016), as well as the SAD detections in highly perturbed fan regions (see 

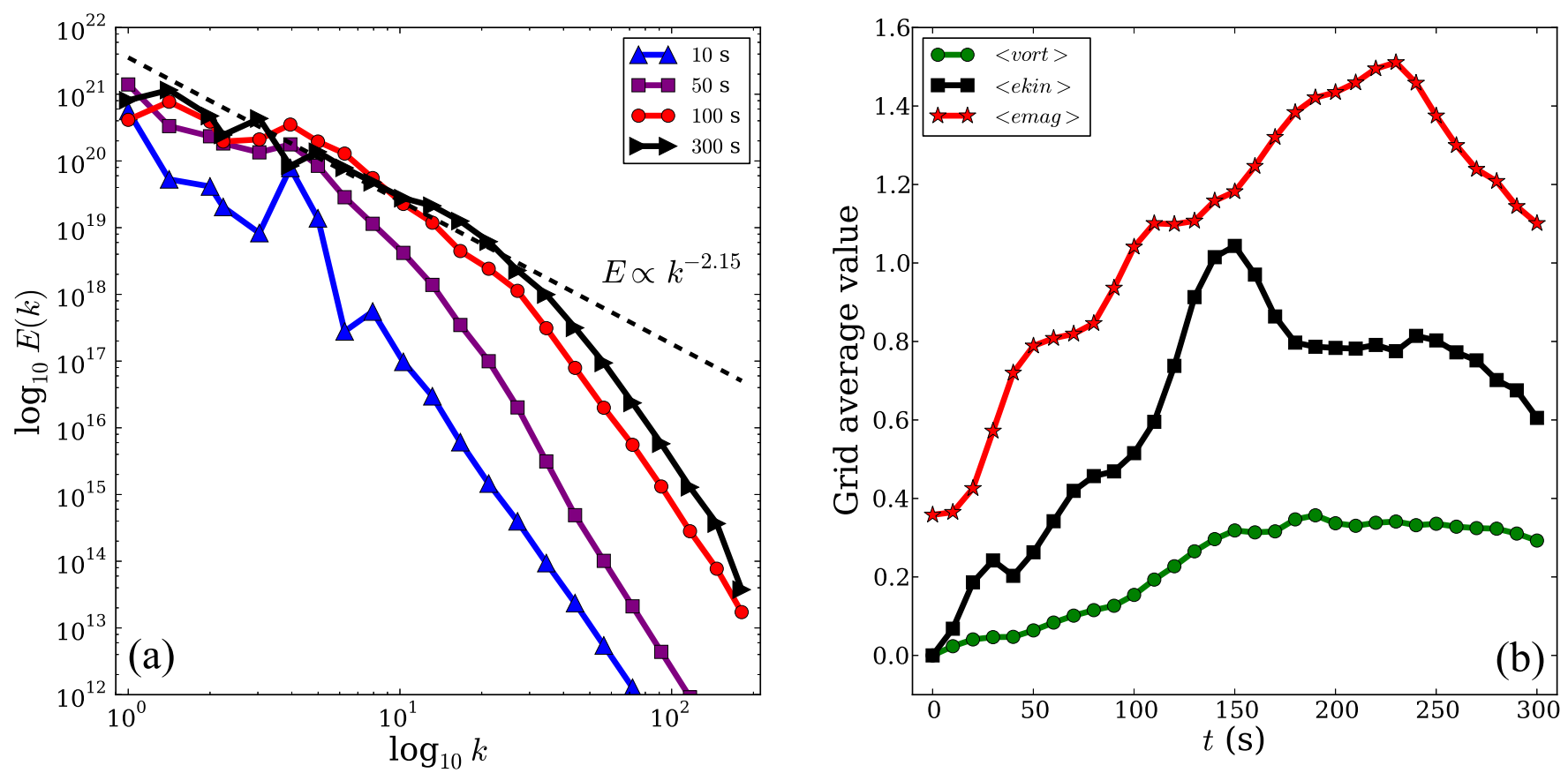

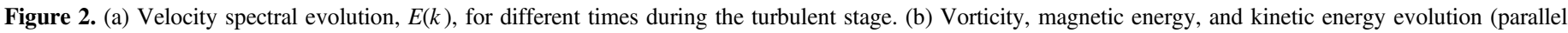
magnetic field case).

Savage et al. 2012b movies), we may expect that a turbulent fan is the natural site where SADs develop.

To generate a statistically stationary turbulent state, we force the low-wavenumber (i.e., large-scale) velocity components by artificially injecting energy. The energy cascades down systematically to smaller scales until it is finally dissipated by viscous action at the smallest scales. Starting from an initial condition, a statistically steady state is achieved after some time in which the average rate of energy added to the system balances with the average energy-dissipation rate (Eswaran \& Pope 1988). To force the system, we use a numerical device that emulates a stirring process.

In order to generate a proper turbulent background, we make use of the Stir unit (FromFile implementation) in the FLASH code (Federrath et al. 2010). The Stir unit allows us to drive a solenoidal force that is calculated in Fourier space and then added as a source term to the momentum equation. This force is represented by $f_{\text {force }}$ on the right-hand side of Equation (2).

The turbulence is driven in a range of wavenumbers that have a parabolic distribution that covers scales from roughly one-third of the computational box $(k=3)$ to the whole computational box $(k=1)$, peaking at $k_{\text {peak }}=2$. The driving amplitude $\left(e_{\text {inj }}=2.0 \times 10^{10}(\mathrm{erg} / \mathrm{s})^{1 / 2}\right)$ was chosen so that the mean temperature after the turbulence reaches a quasi-steady state does not exceed $7 \mathrm{MK}$. The autocorrelation timescale $t_{\text {corr }}$ of the forcing, which is equal to the dynamical timescale of the vortices excited, is $t_{\text {corr }}=L_{\text {peak }} / v=40.1 \mathrm{~s}$, with $L_{\text {peak }}=2 \pi /$ $k_{\text {peak }}$, and an arbitrarily chosen $v=c_{s} / 2$. We carry out the forcing in a pure solenoidal mode (divergence-free), and for the time between successive driving patterns the recommended $10 \%$ of the correlation time is used, i.e., the number of driving patterns $n_{\text {patt }}$ used during runtime is $n_{\text {patt }}=10 t_{\text {turb }} / t_{\text {corr }}$, where $t_{\text {turb }}=7.5 t_{\text {corr }}=300 \mathrm{~s}$ is the turbulent stage duration.

In a nutshell, starting from a homogeneous rest a stationary turbulent state is generated, considered as the initial condition for the fan medium. Figure 2(a) shows the Fourier power spectrum of the velocity field $E(k)$ for different evolution times during the stirring stage. Figure 2(b) shows the grid cell average value of the vorticity, kinetic energy, and magnetic energy against time for the stirring runtime. At early times in this stage the kinetic energy and vorticity have low average values, while the velocity power spectrum reveals that large scales (low wavenumber) are excited but small scales (high wavenumber) are not yet perturbed (blue curve in Figure 2(a)). That is, a stationary state has not been reached. Later on, the energy cascade occupies its entire inertial range, and the system reaches a stationary state. After this, the velocity power spectrum and the other average quantities become stable, with only small oscillations around mean values. Since no additional energy sinks (other than numerical dissipation) were considered and periodic boundary conditions were used, at the beginning the total energy increases monotonically (almost linear) with time. In the stationary state, we find that the power spectrum of the velocity scales as $E(k) \propto k^{-2.15}$ within the inertial range, which is in rough agreement with the expected scaling of a 2D Kolmogorov-like turbulence $\left(E(k) \propto k^{-8 / 3}\right){ }^{6}$ We take as the numerical inertial range $k_{5} \lesssim k \lesssim k_{15}$, adopting the criterion followed by Federrath et al. (2010, and references therein).

When the stationary state is achieved, the kinetic, vorticity, and magnetic functions cease to grow. This stationary state is generated for two initially different magnetic field configurations: a homogeneously parallel one (Figure 2(b)) and an antiparallel one (with respect to the radial direction from the Sun). The antiparallel case defines the position of the CS. As the $\mathrm{CS}$ is expected to be a very thin region $(\sim 1 \mathrm{Mm})$ surrounded by the fan itself and vortical features were apparently detected all over the fan region (McKenzie 2013; Scott et al. 2016), we assume that eddies must be formed

\footnotetext{
6 Kolmogorov turbulence corresponds to turbulence in an incompressible unmagnetized medium, which is not the case for the solar corona. The fact that we have such a spectrum stems from the fact that shear Alfvén modes (which are incompressible) dominate the cascade (Goldreich \& Sridhar 1995).
} 

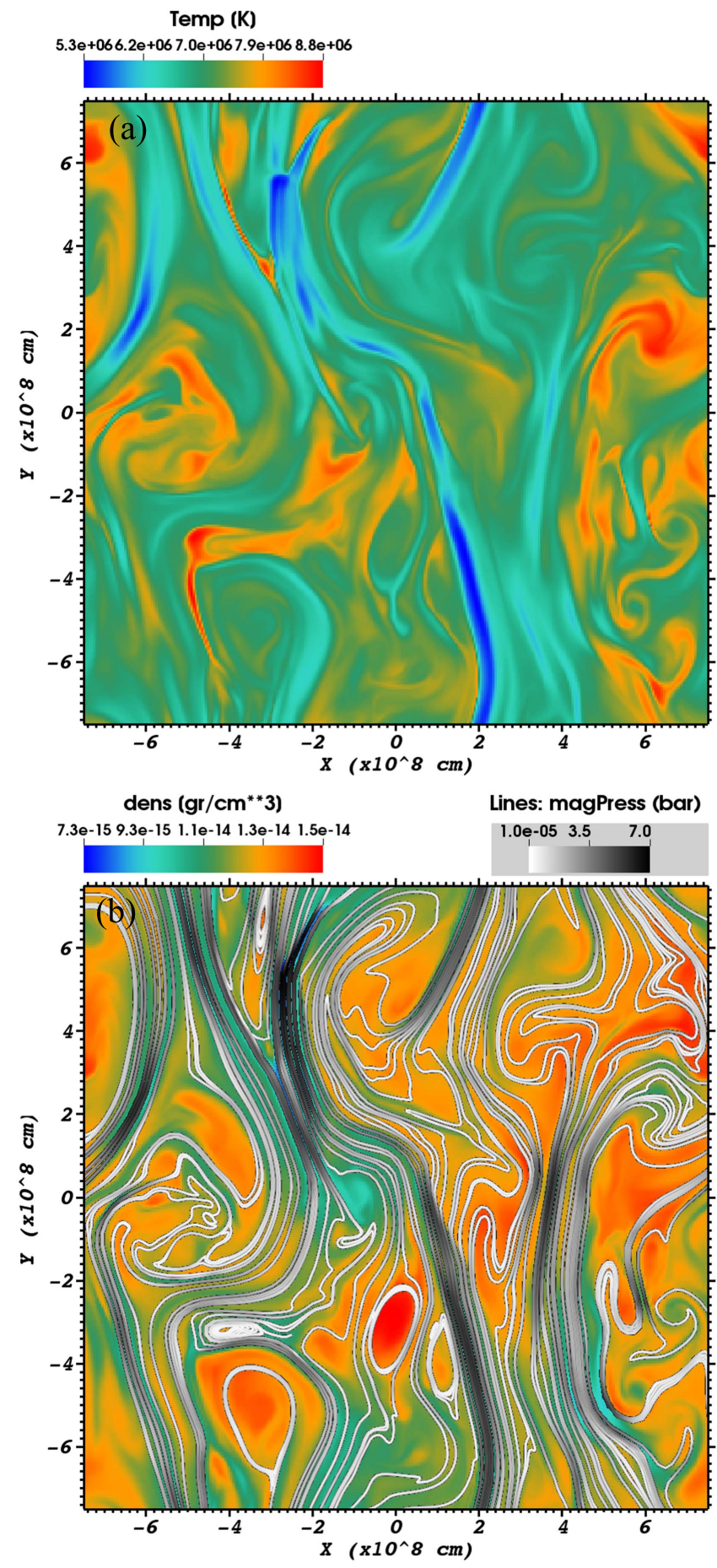

Figure 3. (a) Temperature once the turbulent stage ends, at $t=300 \mathrm{~s}$. (b) Same as (a), but for the density superimposed with magnetic field lines. This is the initial condition for the fan stage.

independently of the location of the CS. Thus, we perform the analysis using the more general parallel configuration, and we will come back later to the discussion of the antiparallel configuration.

\section{RESULTS AND DISCUSSION}

Once the stationary turbulent state is established, we turn off the forcing mechanism, corresponding to the initial conditions of the fan stage. Figure 3(a) displays the density with overlapped magnetic field lines, and Figure 3(b) shows the temperature for the stationary state that is obtained ensuring a final temperature of $\sim 7 \mathrm{MK}$, the desired SAD framework temperature.

At this time, the magnetic topology forms different structures, strands, and vortices. Strands are accumulations of magnetic field lines generally associated with relatively low internal values of temperature and density, meaning that the low gas pressure is compensated by large values of the magnetic pressure (McKenzie 2013). Vortices tend to possess closed magnetic topologies of approximately homogeneous internal density and temperature. The high-density regions, partitioned by the magnetic field lines, correspond either to relatively high or to relatively low temperature. Also, islands (regions with totally closed magnetic field lines) are formed. As in McKenzie (2013) and Scott et al. (2016), the fan has an average initial state value $\beta \gtrsim 1-28$. With this value of $\beta$ the turbulence is almost hydrodynamic, as the magnetic forces do not play a dominant role in the dynamics.

Then we turn on the anisotropic TC. As the vortical regions are the candidates to thermally isolate possible SADs, we apply pressure pulses close to vortices. For simplicity we are not simulating the entrance of SADs to the fan. We assume that as SADs propagate into the fan, their motions are such that they become wrapped up by the magnetic field lines, or, eventually, local reconnections occur inside the fan when two nonparallel field lines approach close enough.

The instantaneous pressure pulses are applied at time $t=305.9 \mathrm{~s}$. Figure 4 shows (i) the temperature pattern and (ii) the density with overlapped magnetic field lines, at an early time $t=312 \mathrm{~s}$. The resulting pulse patterns are labeled by $(A)$, $(B),(C)$, and $(D)$. Figures $4(\mathrm{c})-(\mathrm{d})$ show the same evolved patterns 1 minute later, at $t=372 \mathrm{~s}$, for the temperature with temperature contours superimposed (iii) and the EM (iv). Associated with Figure 4(c), we include an online animated figure for the temperature pattern during the fan stage. The EM contrast of the SAD candidates will fade away fast and before the desired time if during their evolution they are not sufficiently isolated by the magnetic field. In other words, to prevail for a significant time span, they need to be included inside an island. This is not the case for features $(B),(C)$, and $(D)$, which cannot be identified as SADs, according to our requirements. Particularly, only vestiges of feature $(B)$ remain, and a slightly hot subdense lane of feature $(D)$ can be appreciated at $x \approx 4 \times 10^{8} \mathrm{~cm}$. Seeing the contour curves enclosing the $\mathrm{SAD}$, note that the temperature gradient is perpendicular to the magnetic field lines, thermally isolating the SAD structure. The different resulting dynamic evolutions of the bursty reconnections, $(A)-(D)$, necessarily reside in the degree of thermal isolation that the magnetic topology provides during their time evolution.

Figure 5 shows an EM cut over cavity $(A)$ (dotted line in Figure 4(d)) at times $t=312,372$, and $398 \mathrm{~s}$, respectively. The totally enclosed cavity $(A)$ is maintained over time with an EM contrast great enough to allow an SAD observation (see Figure 4(b) in Savage et al. 2012b).

As mentioned, we implemented an external mechanism to develop the turbulent state based on an observationally established turbulent fan description (Savage et al. 2012b; McKenzie 2013; Scott et al. 2016). While not conclusive, SAD detections mostly occur during the decay phase of the flaring activity. Thus, the artificial forcing turnoff could be in line with 

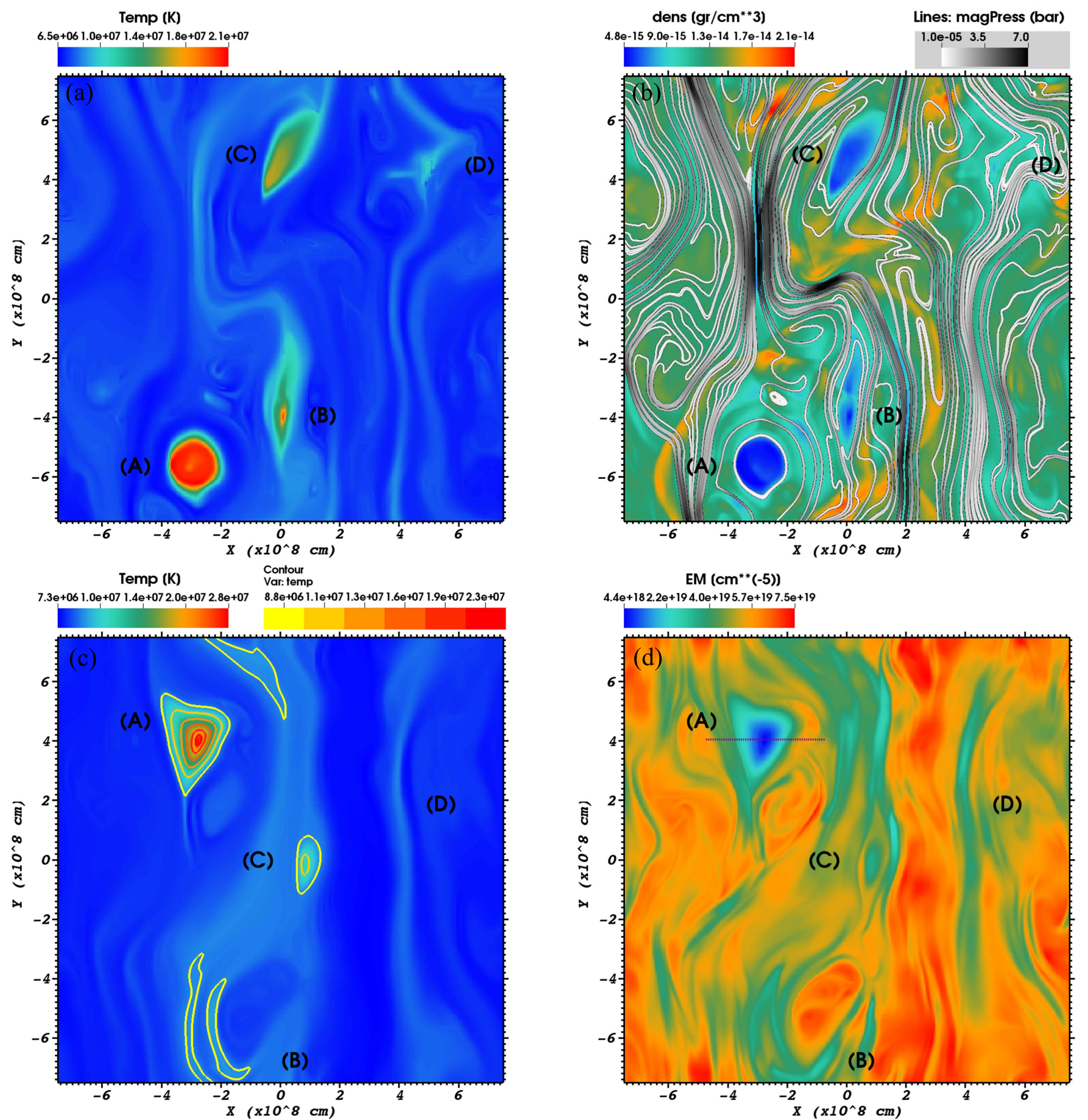

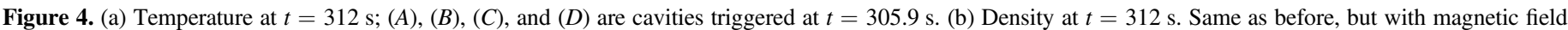

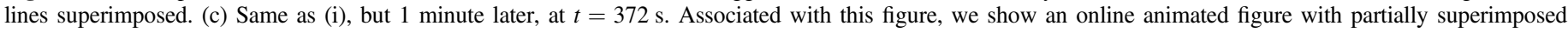

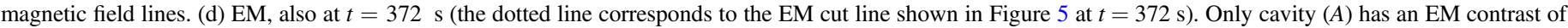
$\gtrsim 4,(B)$ scribbled, $(C)$ smoothed out, and $(D)$ faded away. (Parallel magnetic field case.)

(An animation of this figure is available.)

the flaring decay, leading the system to a decaying turbulent regime with magnetic field lines straightening due to the action of the magnetic tension. At the same time, in the framework of our scenario, downward velocity shears are necessary to explain the motion of the observed low-density regions that can be associated with the action of jets and outflows coming from upper CS structures (Savage et al. 2012a). Shears usually result in $\mathrm{KH}$ instabilities that favor reconnection and consequently the production of isolated islands within the fan (Nakamura et al. 2008). While shears are a model condition to account for SAD velocities, we are now interested in the generation and survival of island topologies by $\mathrm{KH}$ instabilities as this seems to be an important factor for SAD observability.

Nakamura et al. (2008) studied the coupling between MHD scales with $\mathrm{KH}$ vortices and spontaneous magnetic reconnection. They started from a homogeneous $2 \mathrm{D}$ two-fluid plasma as 
the initial condition and found two reconnection types that occur associated with $\mathrm{KH}$ vortices. One type is related with inplane perpendicular magnetic fields across the velocity shear layer. The other is driven when the velocity shear is strong enough to produce highly rolled-up vortices and highly stretched inner field lines, leading to the formation of reconnected islands. They also found that reconnection is triggered even for small values of the Alfvén Mach number $\left(M_{\mathrm{A}}\right)$ of the shear, i.e., $2<M_{\mathrm{A}}<5$. A difference with these authors is that our initial condition is an already-turbulent inhomogeneous one-fluid plasma state with a shear $M_{\mathrm{A}} \simeq 1.3$. For simplicity we have not included a physical resistivity term in the simulations, but we assume that the numerical diffusion that artificially reconnects close field lines provides the necessary reconnection (it actually overestimates the reconnection as we are not able to reach the diffusion scale lengths). To

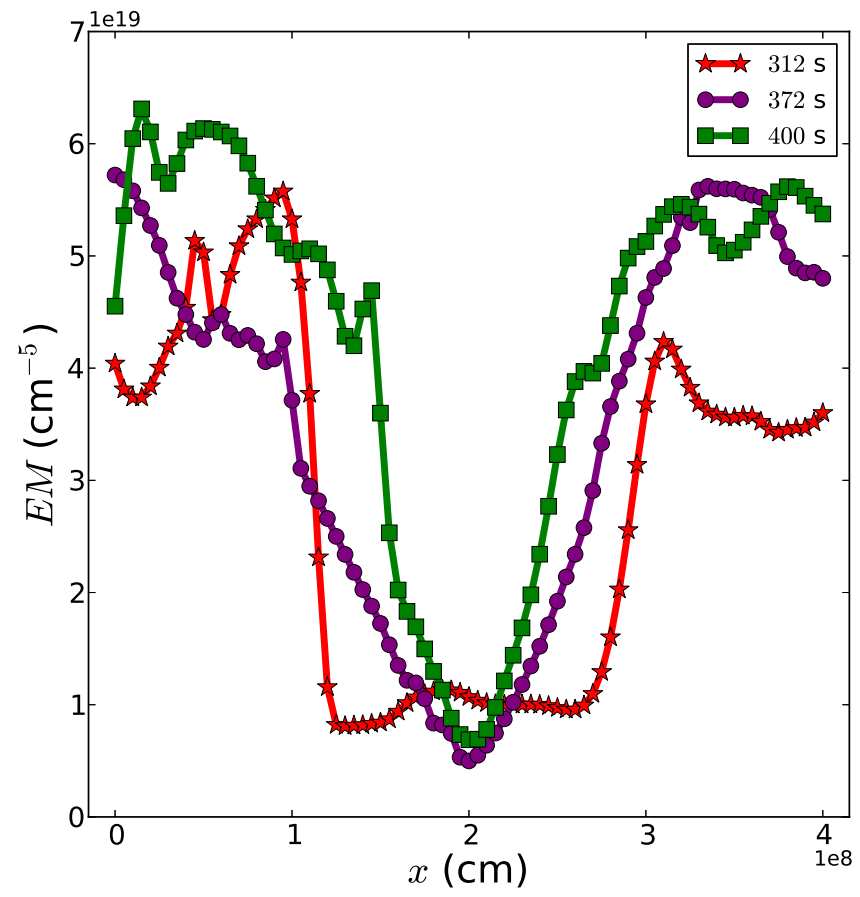

Figure 5. Cut of EM contrast of cavity (B) at times $t=312 \mathrm{~s}, t=372 \mathrm{~s}$ (dotted line in Figure 4(d)), and $t=398 \mathrm{~s}$. qualitatively study the coupling between $\mathrm{KH}$ and reconnection leading to an enhancement of island production, we compare the stationary turbulent state (at the beginning of the fan stage, at $t=303.8 \mathrm{~s}$ ) with the immediate state after the shear.

Figure 6 displays the power spectrum (in Fourier space) of the velocity $E(k)$ (i and ii) and the kinetic energy (iii) just before $(t=303.0 \mathrm{~s})$ and after $(t=304.0 \mathrm{~s})$ the shear is applied (Equation (12)). In this analysis anisotropic TC is not considered. Figure 6(a) shows the velocity power spectrum as a function of $|k|$. The instantaneous shear implies a sudden increase of the curve for the smallest value of $|k|$. Still, the action of the stress tensors yields a contribution that propagates rapidly to the diffusion scales, i.e., at large values of $|k|$. One and a half minutes later (at the end of the fan stage, $t=400 \mathrm{~s}$ ), a typical time of an SAD life, these curves are virtually the same (not shown in the figure). The shear results in an anisotropic contribution to the velocity along the $y$-direction; we accordingly find that the contribution of the velocity strength along $k_{x}$ is almost the same for all times of interest (Figure 6(b)), meaning that the differences between the curves of Figure 6(a) are due to this anisotropic and coherent velocity strength. The Fourier transform as a function of $k_{y}$ (not shown) is almost the same as Figure 6(a). Figure 6(c) shows the kinetic energy power spectrum as a function of $|k|$; the differences between the energy contribution of the modes in the cases with and without shear are more pronounced, but still rather small. We speculate that the action of the stress tensor (when the shear is applied) leads to an increase of mode contributions at all scales, but in particular near the diffusion region, increasing the reconnection rate; this is in agreement with Nakamura et al. (2008), who reported an enhancement of island production when shear was present.

If we consider a 3D magnetic configuration scheme (see Figure 1 of Paper I), the setup described by Table 1, and whose results are shown in Figure 4, will represent the face-on view. To also consider the edge-on view, we perform another simulation where the initial magnetic field, instead of being homogeneous, is the CS given by

$$
B_{y}(x)=\left\{\begin{aligned}
B_{0} & \text { if } x<0 \\
-B_{0} & \text { if } x \geqslant 0
\end{aligned}\right.
$$

with $B_{0}=3.0 \mathrm{G}$. The preparation of the fan is performed with the two stages as in the previous case, but in this case the shear
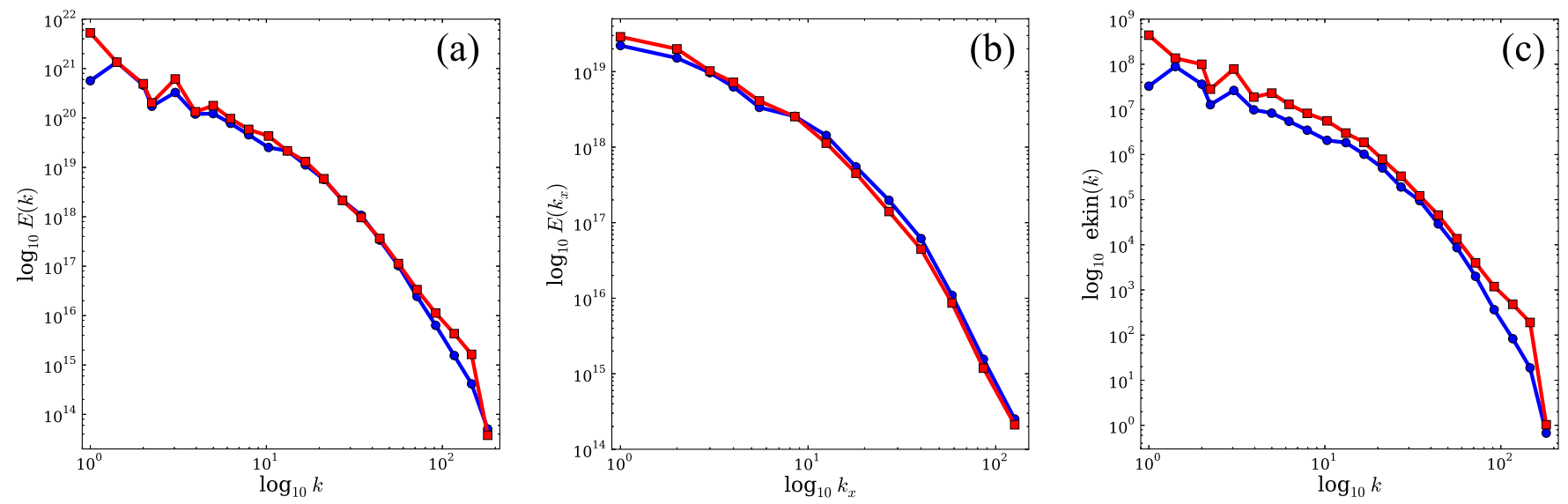

Figure 6. (a) Power spectra of the velocity $E(k)$ as a function of $k$. (b) Velocity $E\left(k_{x}\right)$ as a function of $k_{x}$. (c) Kinetic energy $e k i n(k)$ as a function of $k$. For all cases the red line represents the fan stage at $t=303.0 \mathrm{~s}$ just before the shear is applied, and the blue one just after it, at $t=304.0 \mathrm{~s}$. 

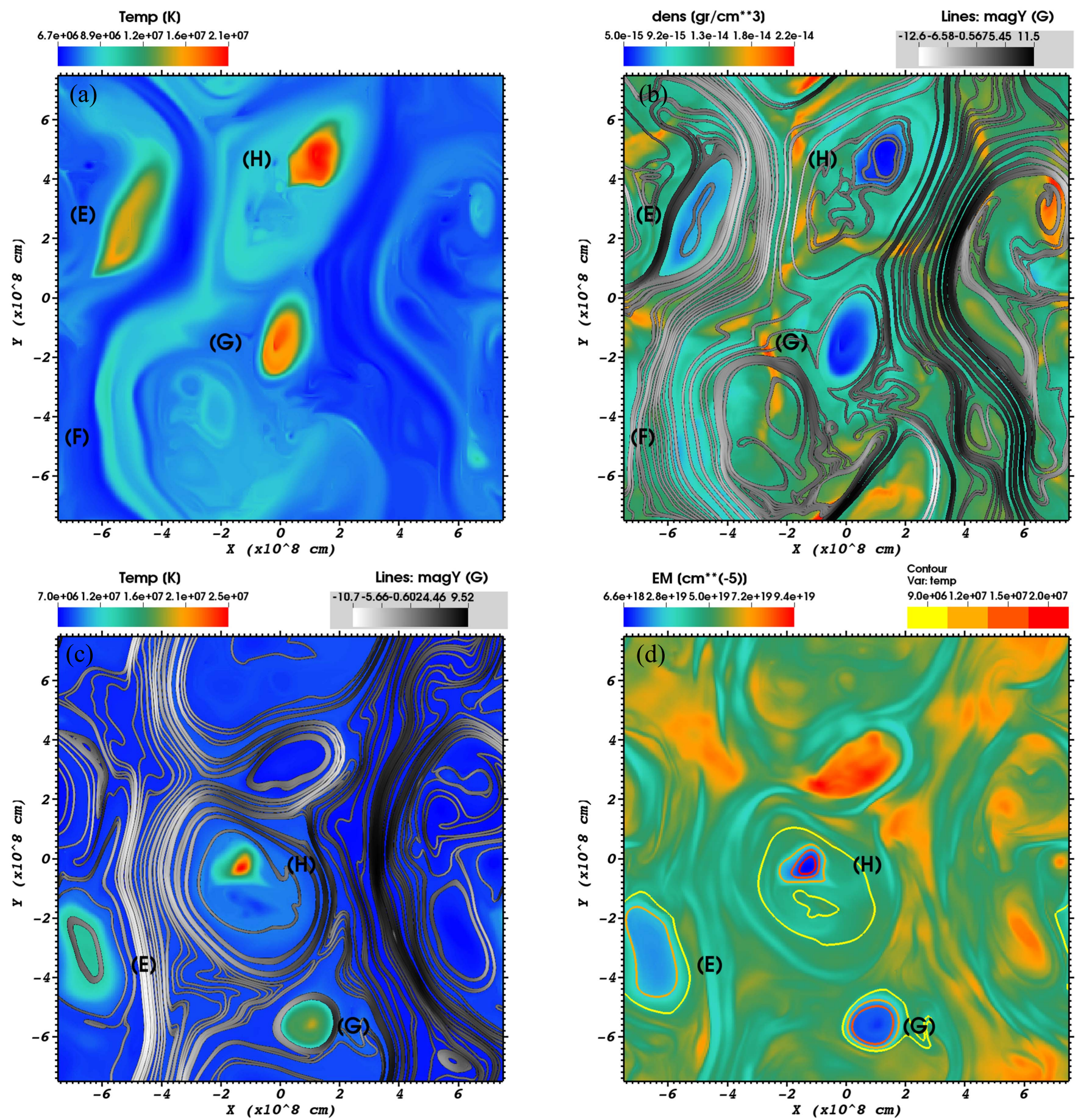

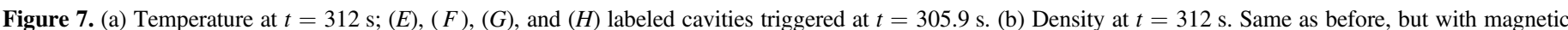

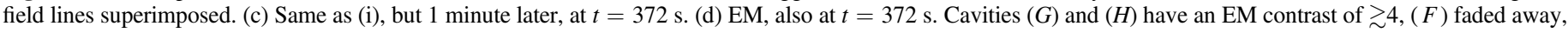
and $(E)$ smoothed out. (Antiparallel magnetic field case.)

(Equation (12)) coincides with the CS location. The instantaneous pressure pulses are again applied at time $t=305.9 \mathrm{~s}$. Figure 7 shows the temperature (i) and the density with overlapped magnetic field lines (ii) at an early time $t=312 \mathrm{~s}$. The resulting pulse patterns are labeled $(E),(F),(G)$, and $(H)$. Figures $7(\mathrm{c})-(\mathrm{d})$ show the same evolved patterns 1 minute later, at $t=372 \mathrm{~s}$, for the temperature with magnetic field lines superimposed (iii) and the EM with temperature contours (iv). Same as before, the EM contrast of the SAD candidates will fade away fast and before the desired time if during their evolution they are not sufficiently isolated by the magnetic field. In fact, the feature $(F)$ triggered in a location where the magnetic field lines are almost straight is not appreciated even at an early time (Figures 7(a)-(b)). The cavities that survive and can be identified as SADs are located mainly in the neighborhood of the $\mathrm{CS}^{7}{ }^{7}$ where the magnetic field is rolled up, forming closed vortices and islands.

\footnotetext{
7 This is noted more clearly when the turbulence stage is carried out using larger wavenumbers compared with those chosen here. See also Paper I.
} 
The observations have generally shown that SADs are distributed along the whole fan and not necessarily concentrated in the CS plane-which may indicate that shears are not always coincident with the CS plane, and that SADs are preferentially detected in face-on views rather than in edge-on ones. Within the current 2D scheme, we conclude that the setups representing the face-on view (Figure 4) and the edge-on one (Figure 7) are both able to produce detectable SADs satisfying the above requirements, where the face-on case seems to be more general and less restrictive than the edge-on one. However, in the 3D scheme the electronic number density also depends on the $z$-direction. Therefore, as the CS is much longer (along the arcade axis) than thicker (perpendicular to the arcade axis), to get an observable SAD, the column plasma (or fan) and the subdense cavity characteristic longitudes must be approximately of the same order (Paper I). Thus, the EM contrast requirement $(\gtrsim 4)$ is much more likely to be satisfied in the face-on view than in the edge-on one.

\section{CONCLUSIONS}

SADs are known to be subdense dark moving trails in turbulent fan regions above flaring arcades. These dynamic features, generally coming from the upper and cooler background corona, twist between fan interstices of inhomogeneous density and magnetic field. Several scenarios have been proposed to account for the nature and dynamical origin of SADs. In previous works we proposed a scenario where SADs are cavities created by the nonlinear waves disturbing the density, and lasting for times comparable with observations. They are triggered by bursty localized reconnection events occurring in the upper background corona or/and in the nearer fan region. The different proposed scenarios have generally eluded the systematic consideration of TC effects of these structures moving in hot and large temperature gradient fans ( $T \gtrsim 7 \mathrm{MK}$ ), which would tend to diffuse the SADs on timescales shorter than their observed lifetime. Here we analyzed different plasma conditions, considering anisotropic $\mathrm{TC}$, that make possible the survival of SADs, avoiding the usually strong thermal damping. We consider SADs as subdense voided cavities with a radius of $\sim 1 \mathrm{Mm}$ (almost the smallest values observed), which can maintain EM contrast values $\gtrsim 4$ and prevail for at least 1 minute in a hot $2 \mathrm{D}$ fan medium. We found that triggered subdense cavities moving in a turbulent vortical magnetized fan can be identified as SAD features if in their downward motion they become enveloped by the magnetic field in such a way that heat conduction is inhibited. We also found that an increase of magnetic island production, where SADs are completely thermally isolated, reinforces the lifetime duration of SADs. Islands are expected due to shears produced by upper observational CS outflows and jets that lead to Kelvin-Helmoholtz instabilities, allowing the reconnection of close enough magnetic field lines. The large fan values of $\beta$ found are in agreement with observations, also reinforcing the turbulence development, i.e., the larger the $\beta$ values, the more efficiently the gas pressure will bend the magnetic field lines. This allows the plasma envelope into highly rolled-up vortices and totally isolated islands, preventing the action of the TC diffusion.

This work was supported by CONICET (Argentina) through a $\mathrm{PhD}$ grant. A. Esquivel acknowledges support from CONACYT (Mexico) grant 167611 and DGAPA-PAPIIT (UNAM) grants IN 109715 and IG-RG 100516. The software used in this work was in part developed by the ASC/Alliance Center for Astrophysical Thermonuclear Flashes at the University of Chicago. We also thank the VisIt team.

\section{REFERENCES}

Asai, A., Yokoyama, T., Shimojo, M., \& Shibata, K. 2004, ApJL, 605, L77 Aschwanden, M. J. 2005, Physics of the Solar Corona. An Introduction with Problems and Solutions (2nd ed.; Chichester, UK: Praxis Publishing Ltd.) Bemporad, A. 2008, ApJ, 689, 572

Cécere, M., Schneiter, M., Costa, A., Elaskar, S., \& Maglione, S. 2012, ApJ, 759,79

Cécere, M., Zurbriggen, E., Costa, A., \& Schneiter, M. 2015, ApJ, 807, 6

Chen, C.-J., \& Lykoudis, P. S. 1972, SoPh, 25, 380

Costa, A., Elaskar, S., Fernández, C. A., \& Martínez, G. 2009, MNRAS, 400, L85

Cowie, L. L., \& McKee, C. F. 1977, ApJ, 211, 135

Eswaran, V., \& Pope, S. B. 1988, CF, 16, 257

Federrath, C., Roman-Duval, J., Klessen, R. S., Schmidt, W., \& Mac Low, M.-M. 2010, A\&A, 512, A81

Fryxell, B., Olson, K., Ricker, P., et al. 2000, ApJS, 131, 273

Goldreich, P., \& Sridhar, S. 1995, ApJ, 438, 763

Guo, L.-J., Huang, Y.-M., Bhattacharjee, A., \& Innes, D. E. 2014, ApJL, 796, L29

Hanneman, W. J., \& Reeves, K. K. 2014, ApJ, 786, 95

Hensler, G., \& Vieser, W. 2002, Ap\&SS, 281, 275

Innes, D. E., Guo, L.-J., Bhattacharjee, A., Huang, Y.-M., \& Schmit, D. 2014 ApJ, 796, 27

Lazarian, A., \& Vishniac, E. T. 1999, ApJ, 517, 700

Lee, D., Deane, A. E., \& Federrath, C. 2009, in ASP Conf. Ser. 406, Numerical Modeling of Space Plasma Flows: ASTRONUM-2008, ed. N. V. Pogorelov et al. (San Francisco, CA: ASP), 243

Linton, M. G., Devore, C. R., \& Longcope, D. W. 2009, EP\&S, 61, 573

Liu, W., Chen, Q., \& Petrosian, V. 2013, ApJ, 767, 168

McKenzie, D. E. 2013, ApJ, 766, 39

McKenzie, D. E., \& Hudson, H. S. 1999, ApJL, 519, L93

McKenzie, D. E., \& Savage, S. L. 2009, ApJ, 697, 1569

Nakamura, T. K. M., Fujimoto, M., \& Otto, A. 2008, JGRA, 113, A09204

Pagano, P., Reale, F., Orlando, S., \& Peres, G. 2007, A\&A, 464, 753

Prialnik, D. 2000, An Introduction to the Theory of Stellar Structure and Evolution (Cambridge: Cambridge Univ. Press)

Savage, S. L., Holman, G., Reeves, K. K., et al. 2012a, ApJ, 754, 13

Savage, S. L., McKenzie, D. E., \& Reeves, K. K. 2012b, ApJL, 747, L40

Scott, R. B., McKenzie, D. E., \& Longcope, D. W. 2016, ApJ, 819, 56

Seaton, D. B., \& Forbes, T. G. 2009, ApJ, 701, 348

Spitzer, L. 1962, Physics of Fully Ionized Gases (2nd ed.; New York: Interscience)

Yokoyama, T., \& Shibata, K. 2001, ApJ, 549, 1160 